\title{
An Edge-Based Moving Object Detection for Video Surveillance
}

\author{
M. Julius Hossain and Oksam Chae* \\ Department of Computer Engineering, Kyung Hee University, \\ 1 Seochun-ri, Kiheung-eup, Yongin-si, \\ Kyunggi-do, Korea, 449-701 \\ mdjulius@yahoo.com, oschae@khu.ac.kr
}

\begin{abstract}
We present a novel approach for extracting moving objects, suitable for intrusion detection and video surveillance systems. Proposed method is characterized by robustness to illumination changes, acclimation to the changes in constituents of background and significantly reduced false alarm rate. We extract pieces of edge information from images and represent these segments with efficiently designed edge classes. Proposed algorithm for matching and updating of edges incorporates the robustness and resilience to intrusion detection system, which is illustrated by the results of our experiments.
\end{abstract}

\section{Introduction}

Automated video surveillance is an important research area in the commercial sector, as many organizations today want to shore up the safety and security of their employees, visitors as well as the premises and assets. The most significant demand from such systems is to isolate the events of potential interest from a large volume of redundant data. However, image data are often contaminated from various unimportant and nuisance noise which contribute unacceptably high false alarm rates. Due to the change of illumination, part of the background may be detected as moving object. The efficiency of surveillance technique lies in the successful detection of the regions with significant changes, ignoring the effect of noise and illumination [1].

We use edge information, which is more robust against illumination changes. Edges are extracted from input image as a unit of segment and a flexible method is proposed for the conformity between two edge segments. Representation of edge segment reduces the affect of noises as noises are found sparse and in a small group of points [2], which is simply ignored in edge extraction step. Reference edges are updated to adapt with the change in background scene as well as to the slow change of illumination. Proposed conformity method between corresponding edge segments of input image and reference image permits the fluctuation of camera focus or calibration error in a certain scale, which helps to reduce the false alarm rate.

\footnotetext{
* Corresponding Author.
} 


\section{Related Works}

A lot of research effort is devoted detecting moving object with various processing steps and core algorithms. Early detection methods were based on difference image, obtained by subtracting the reference image from current input image where the resultant image is thresholded to obtain new objects. Many researchers surveyed and reported experiments on many different criteria to choose threshold value and achieve application specific requirements for false alarms and misses [3,4]. However, determination of an optimal threshold automatically is very difficult, where the applications are sensitive to noise and frequently affected with variations in illumination [1]. Some researchers detect the occurrence of significant change at a given pixel by choosing one of the competing hypotheses describing the intensity change on that pixel [5]. In some statistical approaches [6], the distribution of changes in brightness of reference image and input image are analyzed to discriminate the changes due to illumination variations from those due to new scene contents. It solves part of the problems contributed from illumination change but cannot obtain the complete structure of moving objects. Moving edge extraction method [7] utilizes only edge pixels to reduce the effect of noise and illumination. The performance of a change detection algorithm can be evaluated visually and quantitatively based on application needs. Quantitative evaluation is more challenging, primarily due to the difficulty of establishing a valid "ground truth" [8].

\section{Description of the Proposed Method}

We extract pieces of edge information from images by applying Canny edge extraction algorithm [9] and represent them as efficiently designed edge classes [10] where each segment can be accessed independently. Initially the extracted edge segments form the reference edge list. Input edges are extracted from current image, each segment is searched in the reference edge list and symmetric edges are eliminated. Moving objects are detected from the remaining edge segments, called moving edges. Finally, reference edge list and temporary edge list are updated by changing their weight values associated with them according to their availability in the same position in current fame. Accumulation of moving edges forms temporary reference edge list, which reflects the stability of respective edge. Figure 1 depicts the overall structure of the proposed method.

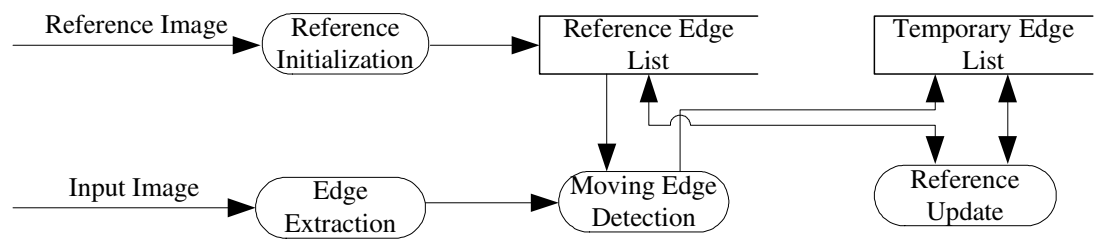

Fig. 1. Block diagram of the proposed method 


\section{Detection of Moving Object}

Proposed method for detection of moving object is depicted in Figure 2. Edges, extracted from current image are not complete due to noise, breaks from non-uniform illumination and other effects that introduce spurious intensity discontinuities. To assemble edge pixels into meaningful edge segment, the characteristic of pixels of currently labeled edges in a small neighborhood is analyzed. All points that are similar according to a set of predefined criteria are linked to obtain edge segments. Each of the extracted input edge segments is searched in reference edge list. If there exist a similar edge segment in the reference list, corresponding edge is removed from current input edges. In this case, weight of the respective reference edge is increased, if the value is less than the predetermined threshold.

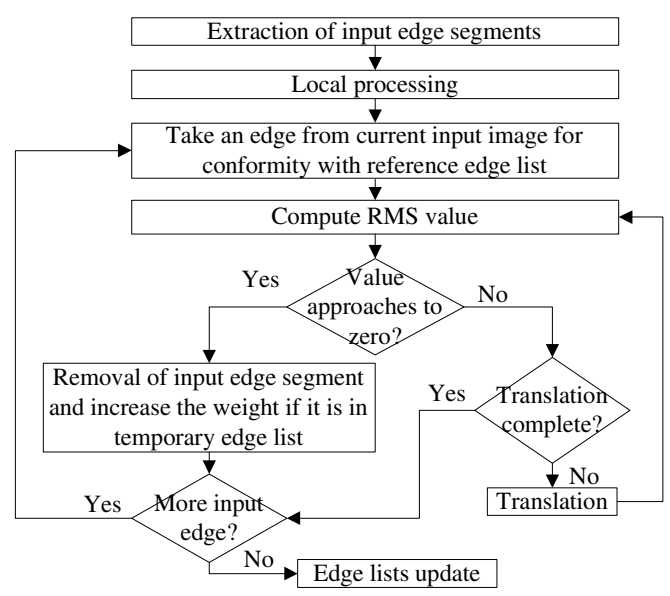

Fig. 2. Detection of moving object

For the conformity, between two edges, we assign a pixel with the value 1 if the pixel belongs to an edge otherwise assign it with 0 . We take the root mean square (rms) value of two edges taken for the matching measure:

$$
M=\sqrt{\frac{1}{n} \sum_{i=1}^{n} d_{i}^{2}}
$$

where $d_{i}$ is the difference of two corresponding pixel values and $n$ is the number of points in the input edge. If the rms value approaches to zero, we consider them as instances of similar edge. Otherwise we translate the input edge and compute the rms value. In case of translation, we choose pixel following the non-decreasing order of city block distance. If these two edges do not match with in predetermined translation area, candidate edges are not considered similar. Translation of input edge in matching process tolerates minor change in camera focus and calibration error; and thus reduces the risk of false alarm.

To adapt the detection process with the change in environment, weight value of each edge segment of both edge lists is updated in every frame. If a moving edge is 
found in next frame at same position, the weight of that segment is incremented else it is decremented. If weight of any edge segment of the temporary edge list reaches specified threshold value, it is moved to the reference edge list from the temporary edge list. An edge segment is eliminated from the temporary edge list if the weight of the segment is zero. In similar fashion, if a reference edge is not found in current frame, the weight of the edge is decreased. The edge is removed from the reference list, if the weight is zero. Figure 5 reflects update in reference edge list and temporary edge list based on the associated weight value. Weight value of a temporary edge varies from 1 to 16 where the range is from 1 to 32 in case of a reference edge.

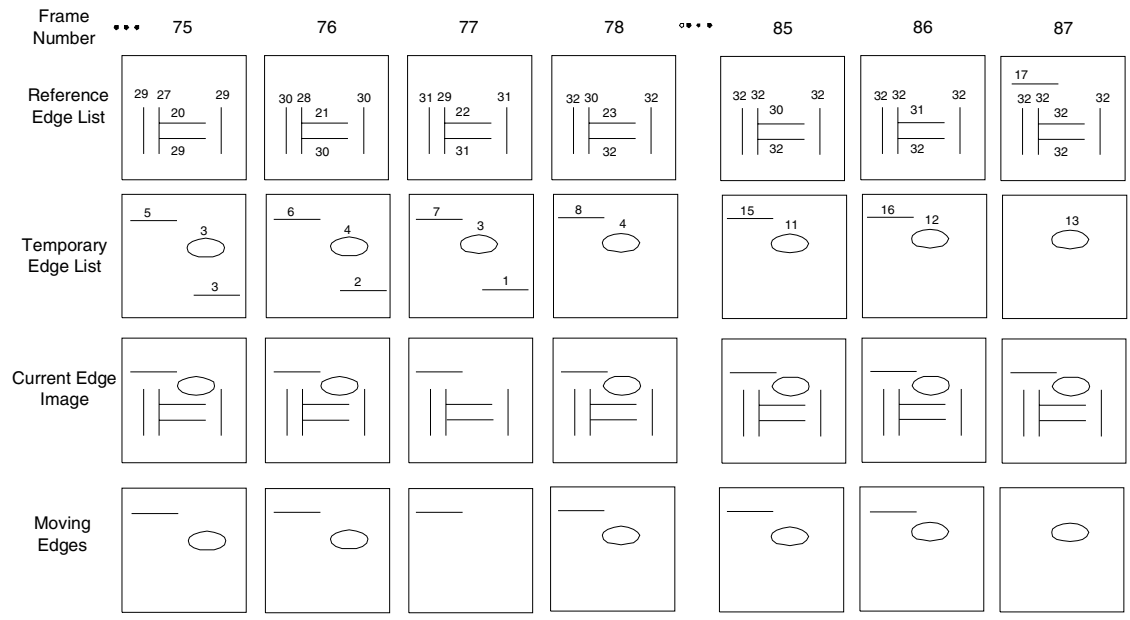

Fig. 3. Update of edge lists

\section{Results}

Proposed method is applied on a sequence of images of size 740 x 560 and intensity range 0-255. We used a system, which included processor of Pentium IV, RAM of 512MB. Visual C++ 6.0 and MTES [11], an image processing environment were used as environment tools. Above system processes 5 frames per second with the application of proposed algorithm and above mentioned environment tools.

Proposed method is adaptive to the change in illumination. Figure 4 shows an instant of reference images. A man is found in figure 5, where the illumination is relatively higher than that of reference image. The difference image is shown in figure 6. Figure 7 shows the edge image obtained by applying Cramer-von Mises approach [12], where they compare the cumulative distribution of the intensities in the two image windows. The result reflects that global thresholding method is not suitable for intrusion detection in dynamic environment. The removal of reference edges by the proposed method results the edge segments of the moving object and detects the intended man, shown in figure 8. Figure 9 to figure 14 reflect moving object detection by proposed method in dynamic background. Figure 9 shows edge image of current reference edge list at frame 84 . Figure 10 is the current image at frame 120 and it 


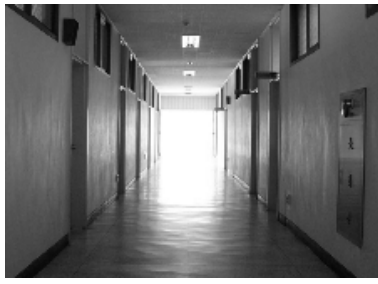

Fig. 4. Background image

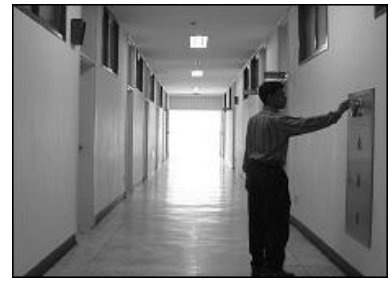

Fig. 5. Current input image in different illumination

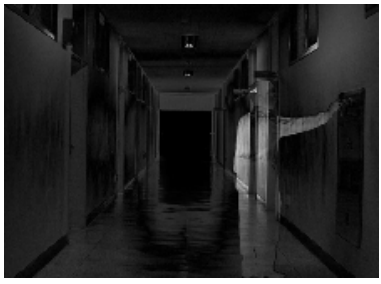

Fig. 6. Difference edge image

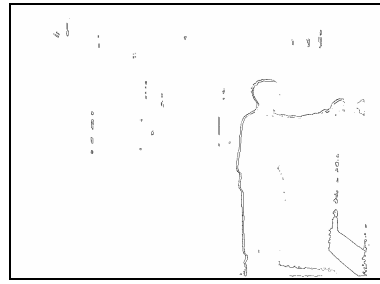

Fig. 7. Detected moving object by CramerVon Mises method

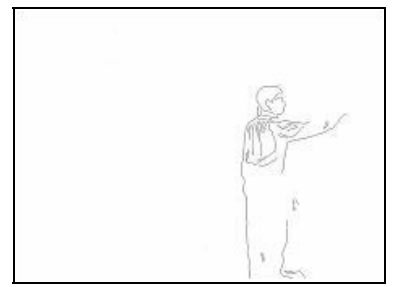

Fig. 8. Detected moving object by proposed method
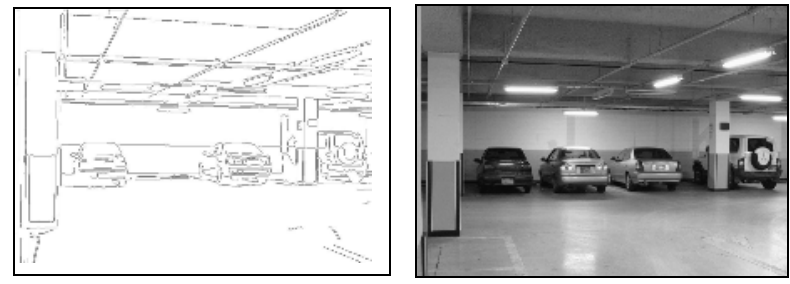

Fig. 9. Edge image of refer- Fig. 10. Input image at frame Fig. 11. Edge image of ence edge list (frame 84) 120

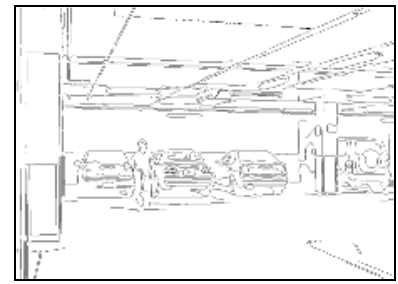

Fig. 13. Edge image of frame 147

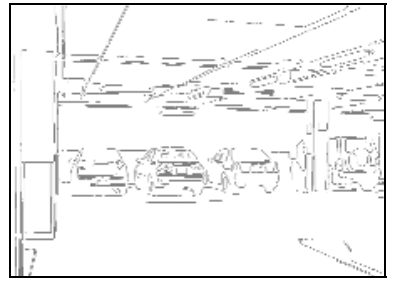

updated reference edge list (frame 136)

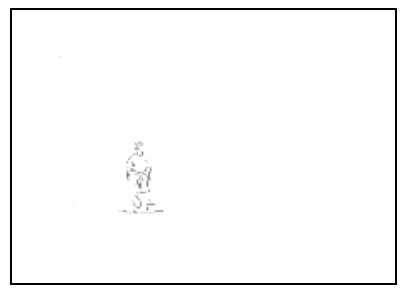

Fig. 14. Detected moving object with updated reference list 
remains unchanged in next 16 frames. So the reference edge list is updated at frame 136 and shown in figure 11. In frame 147, a man is found in the scene, which is shown in figure 12, and corresponding edge image is available in figure 13. The man is detected by conformity and removal of updated reference edge list of figure 11 . The resultant moving object is shown in figure 14.

\section{Conclusions and Future Works}

This paper presents edge extraction based moving object detection using fixed camera, suitable for intrusion detection as well as video surveillance. Proposed method for edge matching and reference update reduces the risk of false alarm due to noise and the change of illumination and contents of background. The proposed method has been tested on numerous real scenes and compared with some existing approaches to justify its effectiveness. However, still there are some scopes to improve the edge-matching algorithm. As a future work, our project pursues to detect moving object from panoramic view of background and to track the moving object.

\section{References}

1. Radke, R.J., Andra, S., Kofahi, O.A., Roysam, B.: Image Change Detection Algorithms: A Systematic Survey. IEEE Transactions On Image Processing, Vol. 14, No. 3 (2005) 294-307

2. Makarov, A., Vesin, J.M., Kunt, M.: Intrusion Detection using Extraction of Moving edges. International Conference on Computer Vision \& Image Processing, Vol. 1 (1994) 804-807

3. Rosin, P.L.: Thresholding for Change Detection. Computer Vision and Image Understanding, Vol. 86 No. 2 (2002) 79-95

4. Smits, P., Annoni, A.: Toward specification-driven change detection. IEEE Transaction on Geoscience and Remote Sensing, Vol. 38, No. 3, (200) 1484-1488

5. Cavallaro, A., Ebrahimi, T.: Video Object Extraction based on Adaptive Background and Statistical Change Detection. SPIE conference on Visual Communications and Image Processing (2001) 465-475

6. Young, S., Forshaw, M., Hodgetts, M.: Image Comparison Methods for Perimeter Surveillance. International Conference on Image Processing and Its Applications Vol. 2 (1999) 799 - 802

7. Hossain, M.J., Lee, J.W., Chae, O.S.: An Adaptive Video Surveillance Approach for Dynamic Environment. IEEE International Symposium on Intelligent Signal Processing and Communication System (2004) 84-89

8. Rosin, P. Ioannidis, E.: Evaluation of global image thresholding for change detection. Pattern Recognition Letter. Vol. 24 No. 14 (2003) 2345-2356

9. Canny, J.: A Computational Approach to Edge Detection. IEEE Transactions on PAMI, 86, (1986) 679-698

10. Ahn, K.O., Hwang, H.J., Chae, O. S.: Design and Implementation of Edge Class for Image Analysis Algorithm Development based on Standard Edge. KISS Autumn Conference (2003) 589-591

11. Chae, O.S., Lee, J.H., Ha, Y.H.: Integrated Image Processing Environment for Teaching and Research. Proceedings of IWIEE (2002) 23-27

12. Sprent, P.: Applied Nonparametric Statistical Methods. Chapman and Hall, London (1993) 